\title{
38. Intracellular Action Potentials of the Guinea Pig Ureter
}

\author{
By Hiroshi IrIsAwa and Makoto Kobayashi \\ Department of Physiology, School of Medicine, Hiroshima University, Hiroshima \\ (Comm. by Y. KUnO, M.J.A.,, April 12, 1962)
}

Although the introduction of the intracellular electrode method provided a great deal of information on the physiology of the smooth muscles, the types of muscles employed in the experiment are still limited to certain muscles such as the pregnant uterus, ${ }^{1,2)}$ the taenia coli $^{\left.3,{ }^{3)}, 4\right)}$ the intestinal muscle ${ }^{5)}$, and the vasa deference. ${ }^{6)}$

In 1942, Bozler ${ }^{7)}$ recorded monophasic action potentials from the ureter of guinea pig, rat and cat, and found that the pattern of action potentials varies from species to species. He also found that the action potential of the ureter shows a delayed repolarization lasting as long as a second like that of the vertebrate myocardium. The finding was later confirmed by Prosser et al. ${ }^{8}$ The ureter has a pacemaker at it's extreme renal end, and the long refractory period which prevents the muscle to contract tetanically. These properties of the ureter muscle are therefore very close to those of the myocardium and appear to be very different from other smooth muscles. It has also been confirmed that in almost all the specimens of the guinea pig, the action potentials of the ureter show the oscillatory potentials superimposed on the plateau phase. These studies have been done through the extracellular recording and it has been desired to have an intracellular recording of the ureter muscle.

The $3 \mathrm{M} \mathrm{KCl}$ filled glass ultramicroelectrodes were prepared by a routine method and the action potentials were recorded with suspended microelectrode according to the method described by Woodbury and Brady. ${ }^{9)}$ The indifferent electrode was made up with a large silversilver chloride electrode and was placed remote in the bathing solution. A high input impedance, negative capacity amplifier was employed. The phenomena recorded were displayed by a cathode ray oscilloscope, and photographed with a Grass type kymograph camera.

Guinea pigs weighing about 500 grams were anesthetized by an intraperitoneal injection of nembutal. The ureter was isolated and cleaned under the dissection microscope in order to remove the surrounding tissue. The isolated preparation has a length over $4 \mathrm{~cm}$ and it was placed in a warm Ringer-Krebs solution. Its composition was following: $\mathrm{NaCl} 0.78 \mathrm{~g} ; \mathrm{KCl} 0.035 \mathrm{~g} ; \mathrm{CaCl}_{2} 0.028 \mathrm{~g} ; \mathrm{NaHCO}_{3} 0.137 \mathrm{~g}$; $\mathrm{NaH}_{2} \mathrm{PO}_{4} 0.0165 \mathrm{~g} ; \mathrm{MgCl}_{2} 0.029 \mathrm{~g}$; water $100 \mathrm{ml}$; dextrose was added shortly before the experiment. The temperature of the bathing 
[Vol. 38,

solution was kept constantly at $37^{\circ} \mathrm{C}$.

The stimulating electrodes consisted of a pair of wick electrodes separated each other about $0.5 \mathrm{~cm}$ in distance. The distance between the stimulating and the recording electrodes was ranged from $0.5 \mathrm{~cm}$ to $1.5 \mathrm{~cm}$. In some instances the distance between the two stimulating electrodes was about $2 \mathrm{~cm}$ and the recording micro-electrode was placed in between them.

The typical patterns of the intracellular potential of the ureter are given in Figure 1. The tracing 1 is the spontaneous action potential. It can be seen that the gradual depolarization precedes the spike potentials. The magnitude of the spikes becomes smaller as the resting membrane level correspondingly decreases. These oscillatory potentials are followed by a slow repolarization phase. The tracing 2 illustrates the result of an electric stimulation, and it can be seen that the patterns of spontaneous and driven action potentials are similar. The tracing 3 is obtained from the other preparation and shows the similar response.

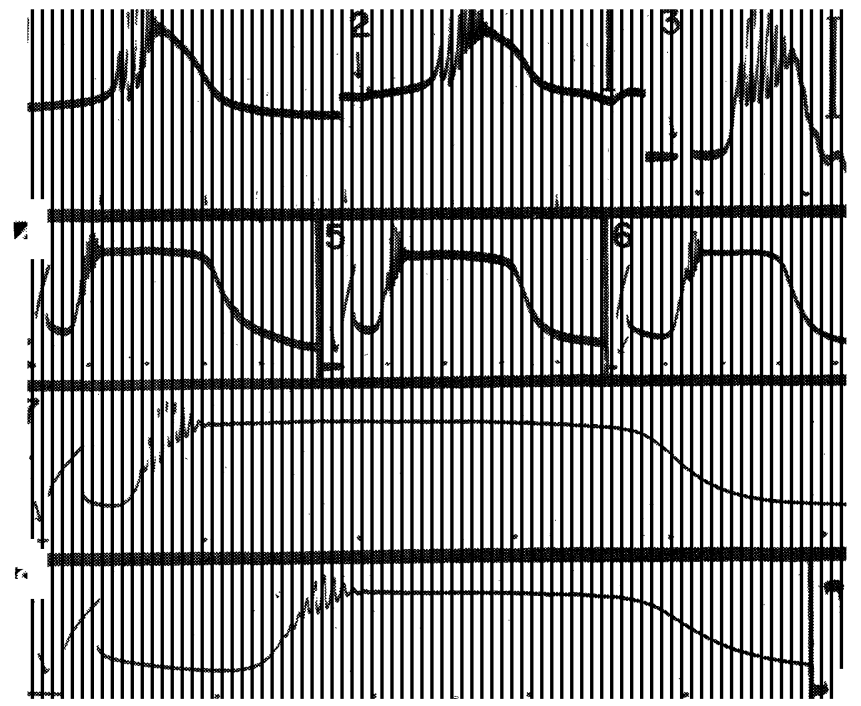

Fig. 1. Typical patterns of the intracellular action potential of the guinea pig ureter. Vertical bars in the tracing 2 (employed for the tracings 1 and 2) and the 3 indicate $20 \mathrm{mV}$. For the tracings 4 to 8 vertical calibration $(50 \mathrm{mV})$ recorded in the right hand of the tracing 8 is employed. Time interval is 2 sec in the top 3 tracings and 1 sec in the others. An arrow shows the onset of the stimulus. See text in detail.

Contrary to these action potentials, the instances which are given in the tracing 4 to 8 show a very long plateau phase lasting as long as over three seconds. The onset of the stimulation is given by an arrow, and the big stimulus artifact indicates the place and the 
duration of the stimulus. After 5 seconds from the complete return of the potential to the resting level in the tracing 5, the second stimulus is applied and the result is illustrated in the tracing 6 . In this tracing, the delayed latency, the smaller size in spikes and the remarkable shortening of the plateau phase are noticed compared to the tracing 5. In order to get a more precise information of the initial part of the action potentials, the experiment is repeated with the more rapid sweep of the recording camera. The tracing 7 corresponds with 5 and the tracing 8 with 6 . It is noticed that although the number of spikes is not much different, the rapid spikes which appear in the third and the fourth of the oscillatory action potential of the tracing 7 are converted into smaller and slower responses in the tracing 8 . It is therefore indicated that the muscle is still in a refractory period even after 5 seconds. Since the resting potential is over 50 to $60 \mathrm{mV}$ in this instance, and the plateau shows a smooth curve, it is assumed that this oscillation and the plateau are not due to the fusion of the successive conducted impulses, or the fusion of the neighboring activities.

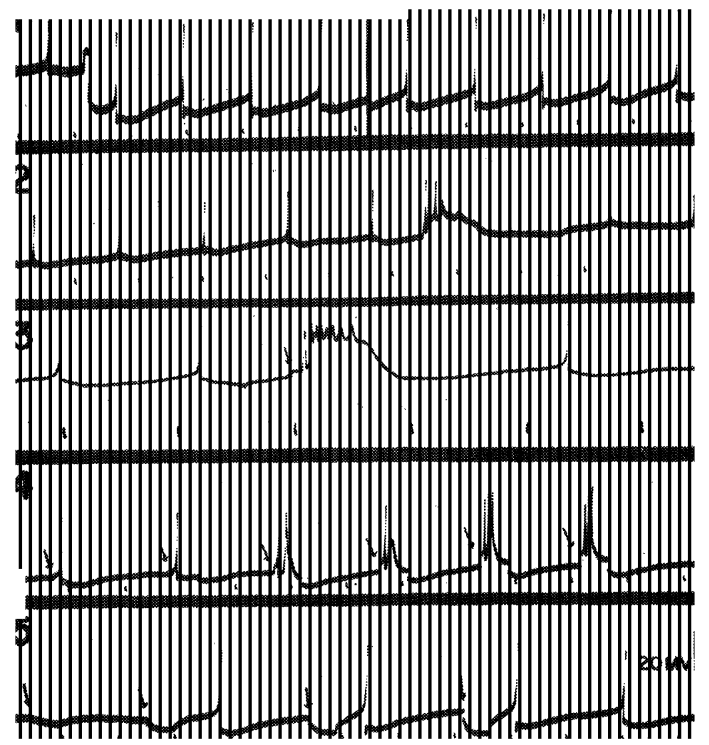

Fig. 2. Spontaneous spike potentials of the guinea pig ureter and the effect of the current intensity on the action potential. Time interval is 2 sec. An arrow indicates the onset of the stimulation.

When the muscle is asphyxiated or left in warm bathing solution as long as two to three hours, very frequently the spontaneous spike potentials can be seen as in Figure 2. We are unable to see the small contractions with these spike potentials. The spike poten- 
tials thus obtained have a frequency of less than 0.5 per second and their magnitude ranged from 20 to $40 \mathrm{mV}$. The pattern of these spike potentials resembles to that of the taenia $\operatorname{coli}^{3), 4)}$ or the uterus. $^{10)}$ The spontaneous repetitious firing such as can be seen in the tracing 2 is often recorded, such repetitive spikes can also be elicited by the supra-threshold electrical stimulation as is illustrated in the tracing 3. The arrow indicates the point of the stimulus.

In the tracing 4 , the cathodal current of a varied intensity and a constant duration ( 1 second) is applied on the ureter, which ceased spontaneous firings. The onset of the current is indicated by an arrow. If the strength of the current is increased gradually, the number of spikes elicited are increased. The increase of spikes soon accommodates as can be seen in the fifth and the last recordings of the tracing 4 . The property of this accommodation appears to be different from the taenia coli. ${ }^{3)}$. The application of the anodal current is illustrated in the bottom tracing. When the current is increased gradually, the spike potential is elicited slightly earlier than the previous one. Still greater increase in the current strength results in the earlier initiation of the spike potential and furthermore the spontaneous response is obtained. It is noted that the stronger the current intensity, the faster the rate of rise of slow depolarization prior to the spikes.

In the present experiment, we have been able to record the long plateau phase of the intracellular action potential of ureter. The mechanisms of this long plateau formation are not yet definitely known. It was very hard to observe a muscle contraction corresponding to the spike potential such as recorded in Figure 2. When the repetitive action potential is recorded, a small contraction can be seen microscopically. The contraction, however, was limited within a very small length, and never conducted all through the preparation. On the contrary, the long plateau is always accompanied by a vigorous and conducted contraction. With this in mind, it is suggested that the plateau has some relation to the excitation of a large number of muscle cell groups, and that the plateau can easily be shortened when the muscle is deteorirated. The intracellular action potential recorded in this experiment shows a remarkably - sinilar pattern to that which has already been recorded monophasically by various extracellular means. ${ }^{7), 11-14)}$ Thus it is suggested that the ureter muscle shows a well "functional syncytium", and a group of the large number of cells is excited simultaneously.

The research reported in this document has been made possible through the support and sponsorship of the U. S. Department of the 
Army through its Far East Research Office, and the Ministry of Education of Japan.

\section{References}

1) Woodbury, J. W., and D. M. Mc. Intyre: Am. J. Physiol., 177, 355-360 (1954).

2) Goto, M.: Jap. J. Physiol., 10, 427-435 (1960).

3) Bülbring, E.: J. Physiol., 128, 200-221 (1955).

4) Holman, M. E.: J. Physiol., 141, 464-488 (1958).

5) Bortoff, A.: Am. J. Physiol., 201, 203--208 (1961).

6) Burnstock, G., and M. E. Holman: J. Physiol., 155, 115-133 (1961).

7) Bozler, E.: Am. J. Physiol., 136, 553-560 (1942).

8) Prosser, C. L., C. E. Smith, and C. E. Melton: Am. J. Physiol., 181, 651-660 (1955).

9) Woodbury, J. W., and Brady, A. J.: Science, 123, 100-101 (1956).

10) Marshall, J. M.: Am. J. Physiol., 197, 935-942 (1959).

11) Greven, K.: Zeit. fur. Biol., 106, 1-15 (1953).

12) Ichikawa, S., and O. Ikeda: Jap. J. Physiol., 10, 1-12 (1960).

13) Burnstock, G., and C. L. Prosser: Am. J. Physiol., 199, 553-559 (1960).

14) Holman, M. E.: Personal communication (1962). 